\title{
The Optimization and Application of the Bowyer-Watson's Triangulation Net
}

\author{
Yang Ding ${ }^{1}$, Fuqi $\mathrm{Chi}^{2}$ and Xianglai Yang ${ }^{1}$ \\ ${ }^{1}$ State Grid of China Technology College, Jinan, Shandong 250002; \\ ${ }^{2}$ Qingdao Yourong Information Science Technology Limited Corporation, Qingdao, Shandong \\ 266555
}

\begin{abstract}
With its basis on discrete points, pressure nephogram is widely used in safety supervision of coal mine. This essay, with the analysis of the problem of the missing "triangular" in the Bowyer-Watson's algorithm, studies and designs a points-adjacent vertex-triangular-based triangulated algorithm, whose function is tested by combining the generating process of pressure nephogram, achieving isoline tracking and the filling of equivalent fields.
\end{abstract}

Keywords: Cloud picture; Delaunay triangulation; Bowyer-Watson algorithms

\section{Bowyer-Watson的三角网生成算法优化与应用}

\author{
丁扬 ${ }^{1}$ ，郗富琦 ${ }^{2}$ ，杨祥来 ${ }^{1}$
}

（1．国网技术学院，山东 济南 250002；2．青岛有容信息科技有限公司，山东 青岛 266555）

摘要: 压力云图在煤矿安全监测中广泛应用，其基础是离散点的三角化。本文通过分析 Bowyer-Watson 算法 “三角形” 丢失问题, 研究设计了一种基于点一邻接点一三角形的三角化算法, 并结合压力云图生成过程对算法性能进行了验证, 实现了 等值线追踪和等值域填充功能。

关键词: 云图; Delaunay 三角网格; Bowyer-Watson 算法

中图分类号：TM 344.1 文献标志码：A

引言

不规则三角网 (TIN) 通过将不规则数据点集进行三角剖分, 使这些不规则点形成连续但不重叠的不规 则三角面片网来拟合连续分布现象的覆盖表面。在矿山压力监测时, 常用 “云图” 颜色展示区域压力强度, 具体包括离散点数据网格化、等值线追踪和等值域填充三个步骤 [1]。其中离散点数据网格化是整个过程 的基础也是本文论述的重点。最常用的离散点数据网格化是 Delaunay 三角剖分法。迄今为止出现了不少 成熟的算法, 如分割 - 合并算法、逐点插入法以及三角网生长法等。其中三角网生长算法由于算法效率较 低, 目前较少采用; 逐点插入法虽然实现较简单, 占用内存较小, 但其时间复杂度差, 效率较低; 分割合并算法最为高效, 但相对复杂, 由于其深度递归, 对内存要求比较高。它们的平均时间复杂度分别有 $0(\mathrm{n})$ 、 $0(n 1 g n)$ 和 $0(n 2)[2]$ 。目前已有许多 Delaunay 三角网格的算法, 比较流行的就是 Bowyer-Watson 算法 $[3,4]$, 国内外多数的研究集中在逐点插入算法的搜索策略的改进 $[5,6]$ 和分治算法与逐点插入算法的合 成 $[7,8]$ 两个方面。本文就 “云图” 生成过程中 Bowyer-Watson 算法所出现的 “三角形丢失” 问题开展 研究, 设计了一种基于点-邻接点-三角形的三角化算法, 并实现了等值线追踪和等值域填充功能。

\section{1 数据结构设计}

一般三角网采用点-边-三角形和点-三角形结构等两种数据结构来实现。点-边-三角形存在检索方便 的优点, 但运算效率直接受三角形废品率 [9]制约, 维护成本较高; 而点-三角形结构所需的存储空间更小。 
本文采用了点-邻接点-三角形的数据结构, 即便于邻接三角形、邻接边的检索又有效控制空间消耗。

（1）离散点表: 离散点表是所有点坐标数据的数据库, 建立唯一标志 ID 便于后续检索, 另外通过邻 接顶点集合存储邻接点替代了 “边” 信息的存储。

class Splash

public Splash(double $x$, double $y$, double $v$, int id)

\{

$\mathrm{X}=x$

$\mathrm{Y}=y$;

$\mathrm{V}=v$;

$\mathrm{ID}=i d$

\}

public Splash(int id)

\{

$\mathrm{ID}=i d$

\}

public double $X$ \{ get; set; \}

public double Y \{ get; set; \}

public double V \{ get; set; \}

/// <summary>

/// 用于标志点的唯一性

I/I </summary>

internal int ID $\{$ get; set; $\}$

/// <summary>

/// 邻接顶点集合

I/I </summary >

internal int[] Adjoin VertexIndexs \{ private set; get; \}

\}

(2) 三角形: 记录最终生成的 TIN 数据, 依赖于离散点表。

class Triangle

\{

I// <summary>

/// V0 顶点索引

I// </summary >

public int v0Index;

/// <summary>

/// V1 顶点索引

I/I </summary>

public int v1Index; 
I// <summary>

/I/ V2 顶点索引

/// </summary>

public int v2Index;

\}

\section{TIN生成算法研究}

\subsection{Delaunay 三角网格与 Bowyer-Watson 算法}

G.L.Dirichlet 提出了一种将平面划分为 Voronoi 区域的方法[10] 设 $\left\{P_{i}\right\}_{i=1}^{n}$ 为平面上一点集, 则每一点 $P_{i}$ 对应一区域 $V_{i}=\bigcap_{1 \leq j \leq n, j \neq i}\left\{P:\left\|P-P_{i}\right\|<\left\|P-P_{j}\right\|\right\}$ 称为点 $P_{i}$ 的 Voronoi 区域（又叫 Thiessen 区域或 Wigner-Scitz 单元), 点 $P_{i}$ 称为 Voronoi 区域 Vi 的形成点。Voronoi 区域 Vi 是互不重叠的凸多边形区域 (含 开口的凸多边形区域), 且 $\bigcup_{i} \bar{V}_{i}$ 覆盖全平面 (如图 1)。我们把 $\bigcup_{i} \partial V_{i}$ ( $\partial V_{i}$ 表示区域 Vi 的边界) 称为 平面的一个 Dirichlet 网格。当点集 $\left\{P_{i}\right\}_{i=1}^{n}$ 给定时, 平面的 Dirichlet 网格是唯一确定的。

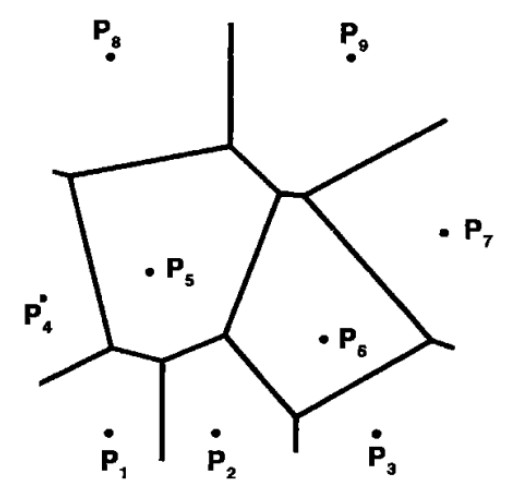

图 1 Dirichlet 网格

当 $\left\{P_{i}\right\}_{i=1}^{n}$ 为非共线点集时, 连接有公共边的 Voronoi 区域的形成点, 得到一链接点的 $\left\{P_{i}\right\}_{i=1}^{n}$ 网格（见 图 2), 称为 Delaunay 三角网格, 网格中的三角形称为 Delaunay 三角形。连接点集 $\left\{P_{i}\right\}_{i=1}^{n}$ 的 Delaunay 三角 网格总是存在的,当不存在退化的情形时, Delaunay 三角网格还是唯一确定的。[9] 


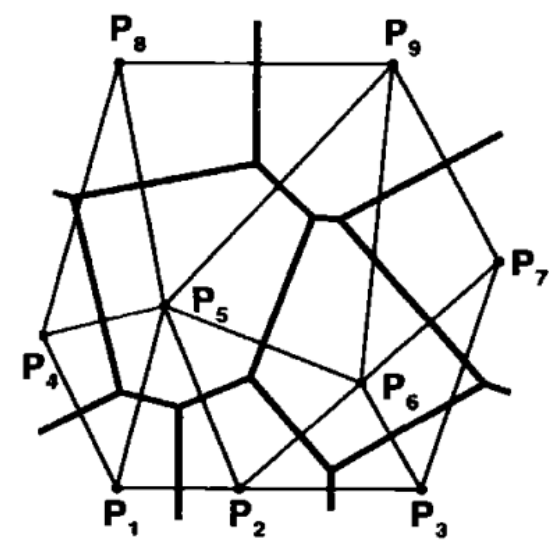

图 2 Delaunay 三角网格

Delaunay 三角网格具有以下性质: 最接近, 以最近临的三点形成三角形, 且各线段(三角形的边)皆不 相交; 唯一性, 不论从区域何处开始构建, 最终都将得到一致的结果; 最优性, 任意两个相邻三角形形成 的凸四边形的对角线如果可以互换的话, 那么两个三角形六个内角中最小的角度不会变大; 最规则, 如果 将三角网中的每个三角形的最小角进行升序排列, 则 Delaunay 三角网的排列得到的数值最大; 区域性, 新 增、删除、移动某一个顶点时只会影响临近的三角形; 具有凸多边形的外壳, 三角网最外层的边界形成一 个凸多边形的外壳。

A.Bowyer 于 1981 年提出了一种生成 Delaunay 三角网格的加点算法, Bowyer-Watson 算法具体实现 如下:

（1）假定已生成了连接若干个顶点的 Delaunay 三角网格;

（2）加入一个新的节点, 找出所有外接圆包含新加入节点的三角形（称为影响三角形, 该三角形的 边称为影响边), 并将这些三角形删除, 形成一个空腔;

（3）空腔的节点与新加入的节点连接, 形成新的 Delaunay 三角形网格;

(4) 调整数据结构 新生成的三角形的数据填充被删除三角形的数据, 余者添加在数组的尾部;

（5）返回第（2）步，直至所有的节点都加入为止。

Bowyer-Watson 算法关键的第 2、3 步图示见图 3:

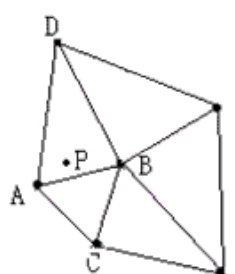

(a)插入新结点P

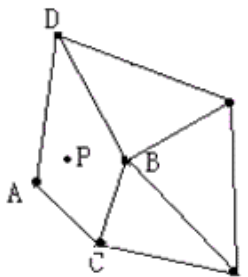

(c)删除边 $A B$

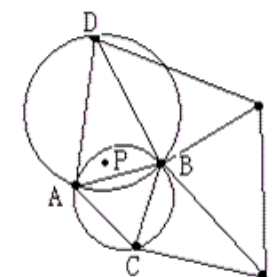

(b) 决定如何连接P与其它顶点

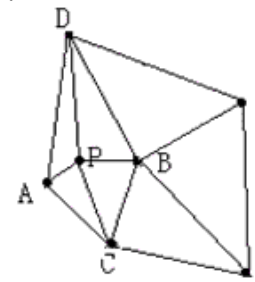

(d)形成三角形

图 3 Bowyer-Watson 算法关键步骤图示 
根据上述 Bowyer-Watson 算法的基本思想，实现如下：

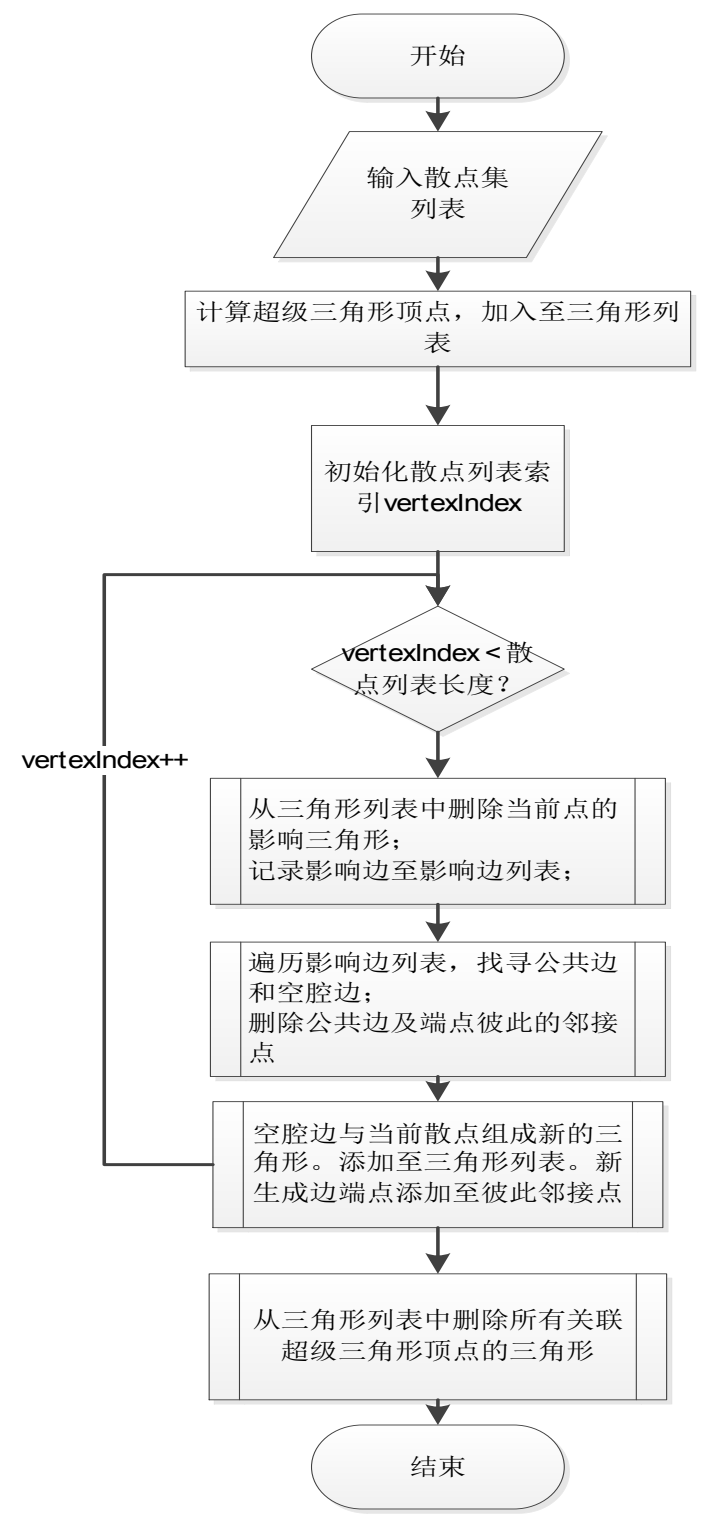

图 4 Bowyer-Watson 算法流程

\subsection{Bowyer-Watson 算法优化}

在散点分布较为均匀的情况下, Bowyer-Watson 算法能较好地生成 Delaunay 三角网格, 但如果数据中 存在狭长散点集合时，使用该算法就有可能导致凸壳边丢失甚至无法形成三角网，存在“三角形丢失”和“狭 长四边形无法形成多边形”现象。

“三角形丢失”现象逐步插入点过程如图 5(a)-(f)所示，经分析，(c)-(e)中三角形 T1、T2、T3、T4 均不 符合 Delaunay 三角网格最优性原则。因此图 5(e)点 4 插入完成后, 执行“从三角形列表中删除所有关联超 级三角形顶点的三角形”时会导致三角形 $\mathrm{T} 3$ 和 $\mathrm{T} 4$ 同时删除, 所以点 $\{0,1,4\}$ 无法形成三角形。 


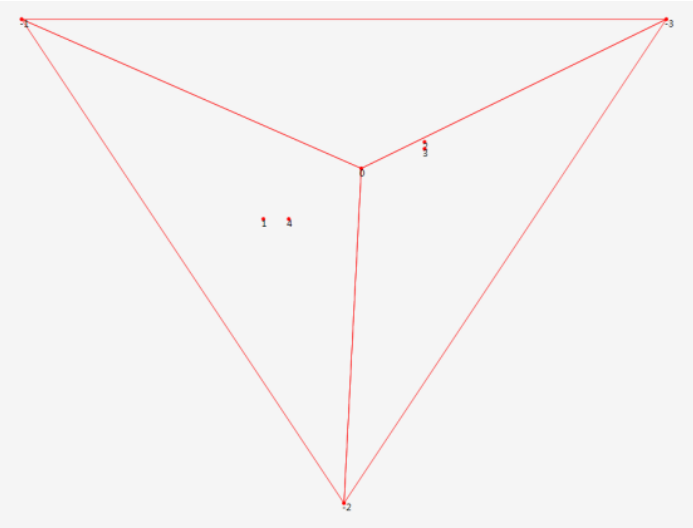

(a)

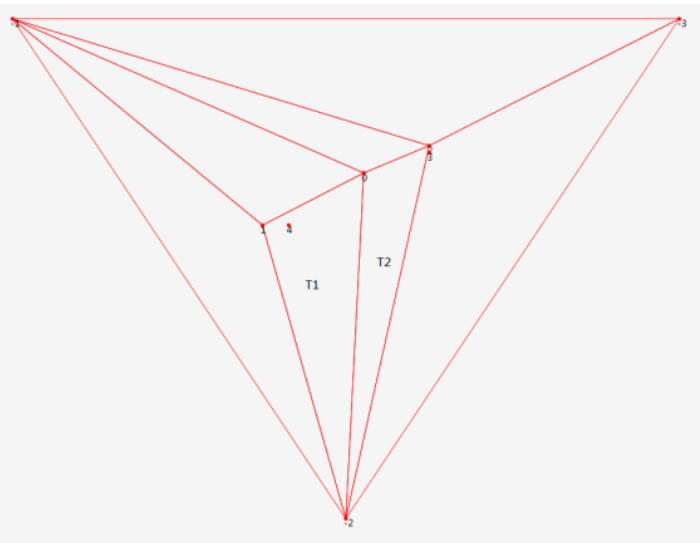

(c)

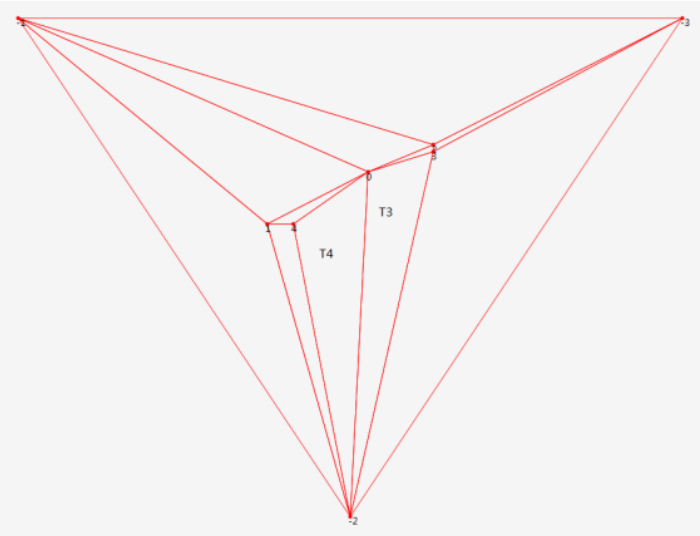

(e)

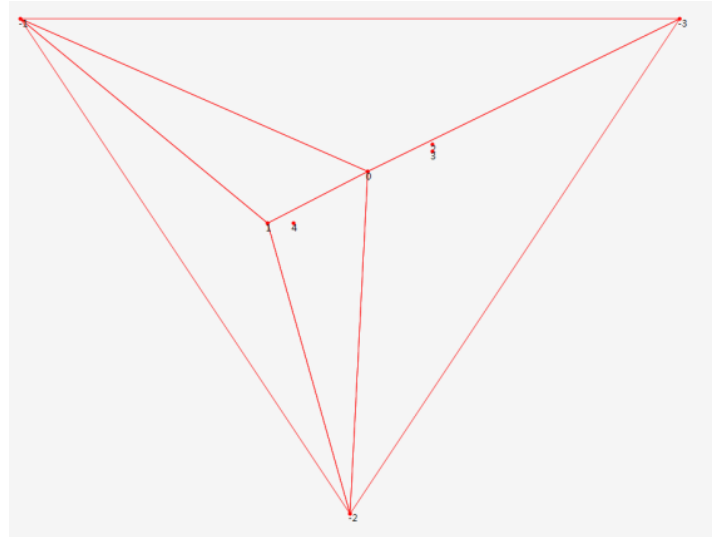

(b)

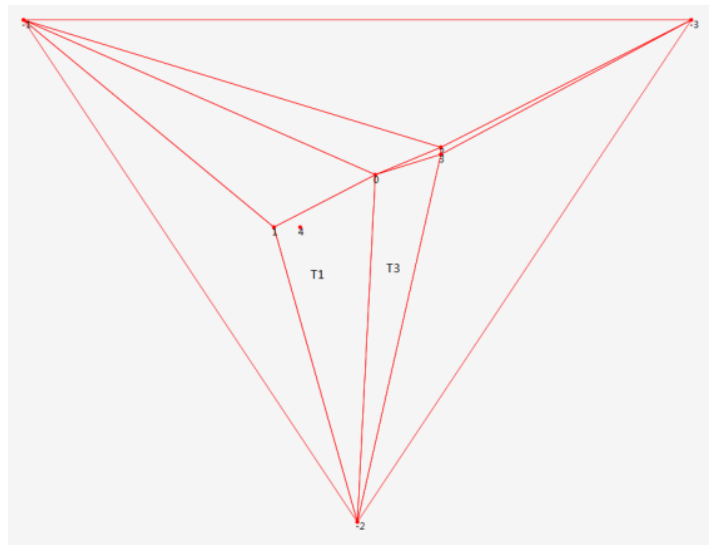

(d)

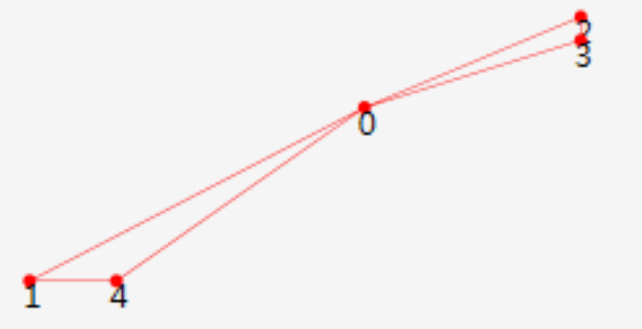

(f)

图 5 “三角形丢失”现象逐点插入过程

“狭长四边形无法形成多边形”现象逐点插入过程如图 6(a)-(d)所示, 其中右侧图片是对左侧散点的放大 效果图。经分析, 图中 $\mathrm{T} 1 、 \mathrm{~T} 2 、 \mathrm{~T} 3$ 均不符合 Delaunay 三角网格最优性原则, 导致无三角形形成。删除掉 所有与超级三角形顶点连接的三角形时会删除所有边。 


\section{ATLANTIS}

PRESS

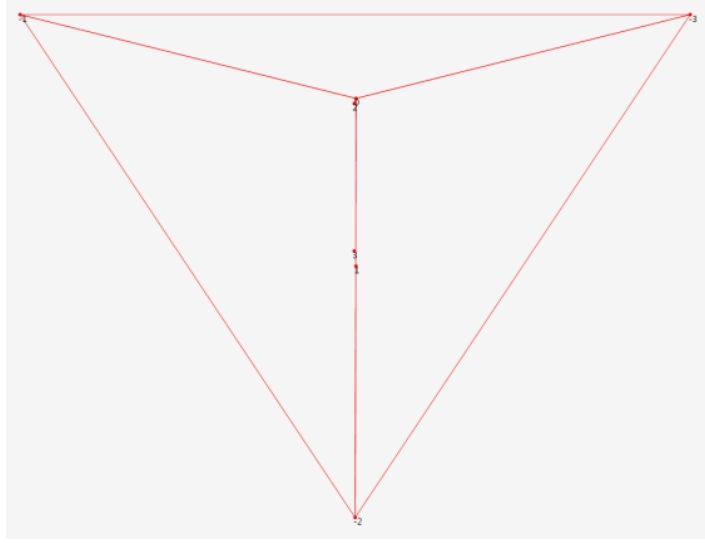

(a)

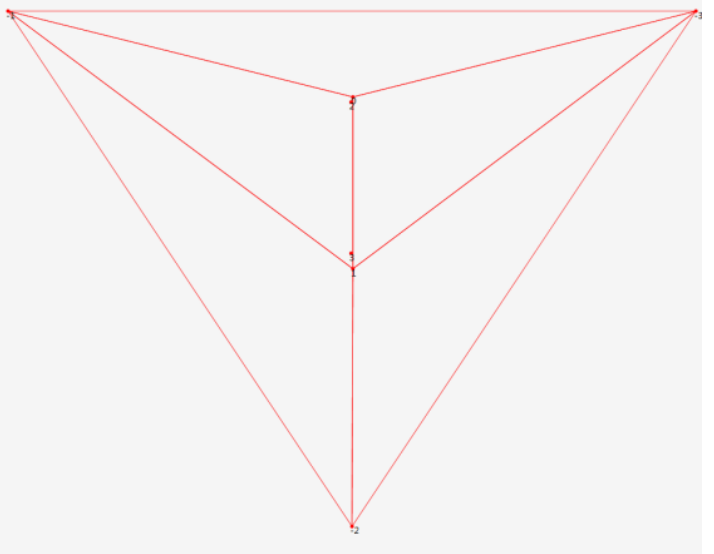

(b)

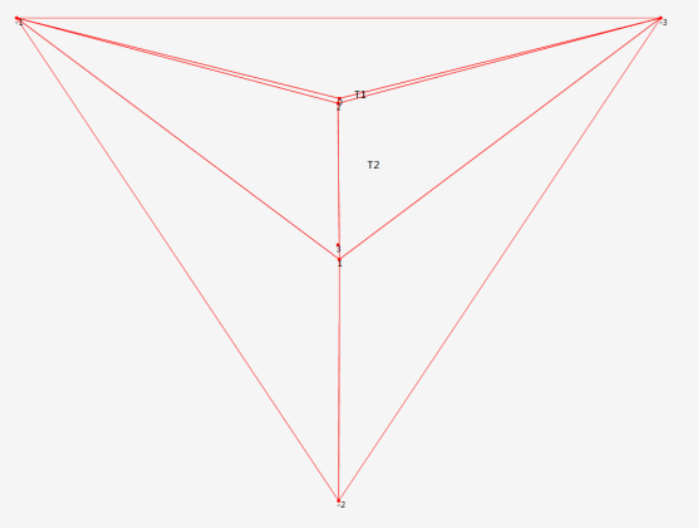

(c)

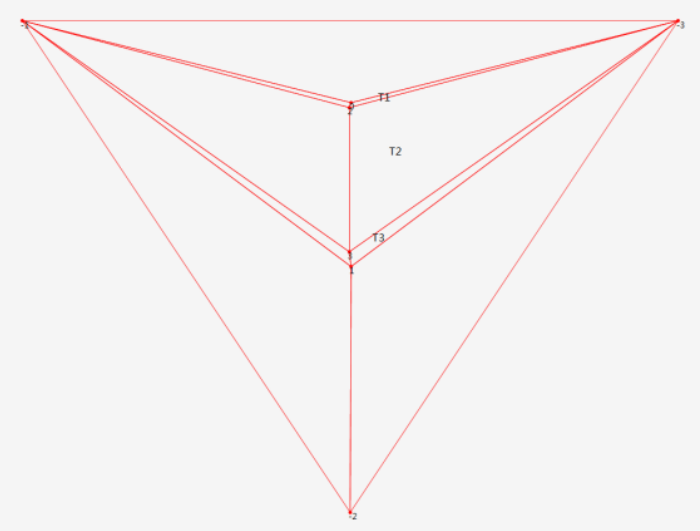

(d)

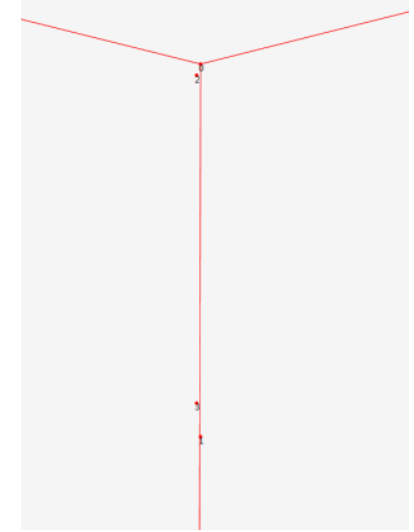

$(\mathrm{a} 0)$

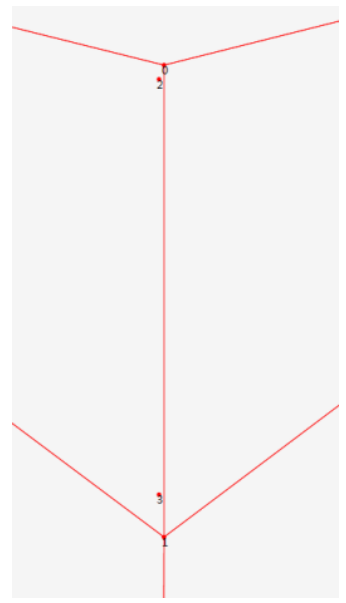

(b0)

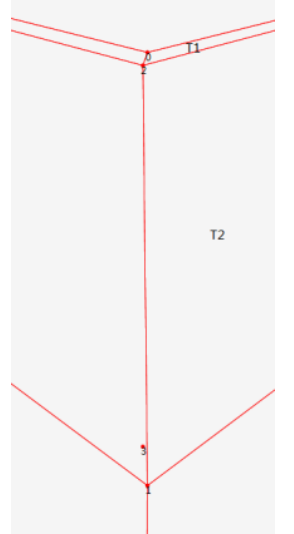

(c0)

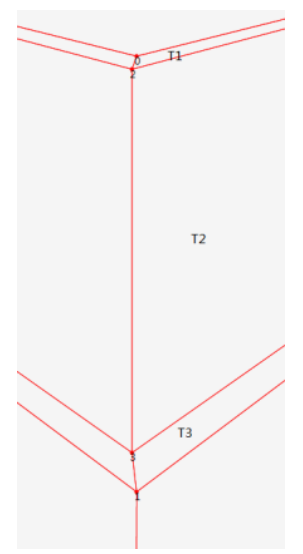

(d0) 


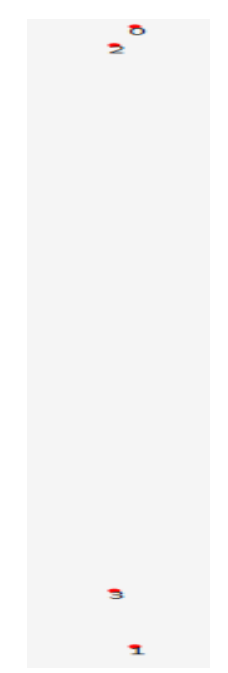

(e)

图 6 “狭长四边形无法形成多边形”现象逐点插入过程

图 5（f）和图 6（6）相对坐标分别见表 1 和表 2:

表 1 图 5（f）相对坐标

\begin{tabular}{|c|c|c|}
\hline 序号 & $\mathrm{X}$ & $\mathrm{Y}$ \\
\hline 0 & 41.7984273088593 & 71.1123717264292 \\
\hline 1 & 28.4143449698921 & 58.3332977294922 \\
\hline 2 & 50.4443511962891 & 77.7778015136719 \\
\hline 3 & 50.4443511962891 & 76.0559856501982 \\
\hline 4 & 31.8826060764624 & 58.3332977294922 \\
\hline
\end{tabular}

表 2 图 6(e)相对坐标:

\begin{tabular}{|c|c|c|}
\hline 序号 & $\mathrm{X}$ & $\mathrm{Y}$ \\
\hline 0 & 113.428571428571 & 490.5 \\
\hline 1 & 113.428571428571 & 40.5 \\
\hline 2 & 110.857142857143 & 477 \\
\hline 3 & 110.857142857143 & 81 \\
\hline
\end{tabular}

为满足最优性原则, 需进行局部最优化处理。Lawson 提出的局部优化过程 LOP(Local Optimization Procedure), 其基本做法如下所示:

(1) 将两个具有共同边的三角形合成一个多边形;

（2）以最大空圆准则作检查, 看其第四个顶点是否在三角形的外接圆之内;

（3）如果第四个顶点是否在三角形的外接圆之内, 修正对角线即将对角线对调, 即完成局部优化过 程的处理。LOP 处理过程如图 8 所示: 

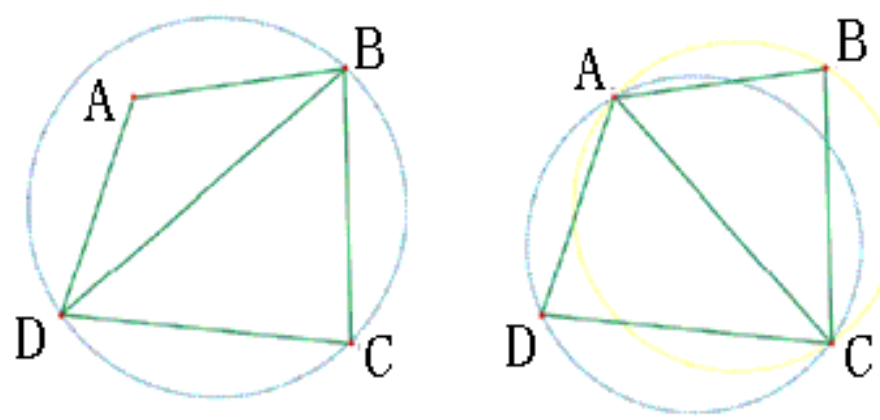

图 8 Lawson 局部优化过程

对所有新生成的三角形遍历其邻接三角形并按照 LOP 方式处理,在形成新的三角形后需要维护新旧邻 接三角形关系, 同时 LOP 新邻接三角形, 该算法运算效率较低, 在工程中不常使用。因此本文提出了一种 基于点-邻接点-三角形的 $\mathrm{O}(\mathrm{nlgn})$ 三角化算法, 其运算效率较高, 可解决实际工程中无法形成三角网和凸壳 边丢失问题。

该算法核心思想是在插入点时计算新生成的三角形是否为固定三角形, 固定三角不再参与后续点的影 响三角形判断, 确保凸壳边不在三角化过程中删除。插入完成所有点并删除带有超级顶点的所有三角形后, 排查是否有遗失凸壳边, 如果存在遗失凸壳边 $\alpha$ (端点 $\mathrm{a} 、 \mathrm{~b}$ ), 通过匹配 $\mathrm{a}$ 的邻接点与 $\mathrm{b}$ 的邻接点的共同 点 $c$, 可以组成新的三角形 $a b c$, 并插入至三角形链表中。算法流程图见图 9。 


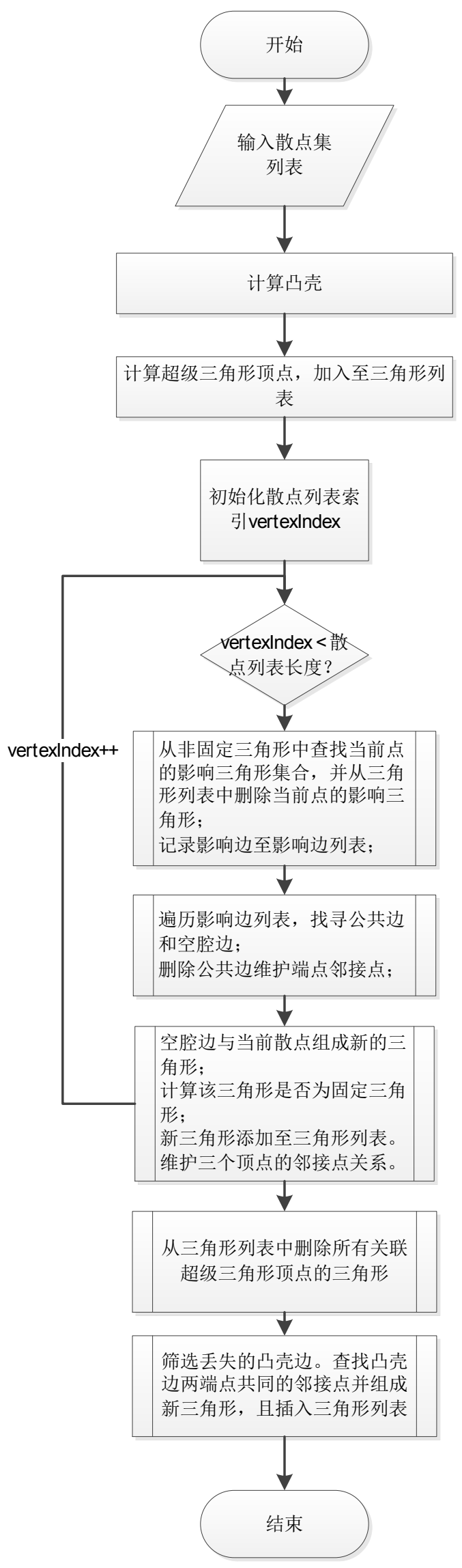

图 9 基于点-邻接点-三角形的三角化算法流程

以上算法涉及相关概念解释如下: 超级三角形, 包含所有散点的三角形; 超级顶点, 超级三角形上的 
顶点; 凸壳, 散点点集合的边界; 影响三角形, 某点在另外三点所组成三角形的外接圆内, 那么该三角形 是某点的影响三角形; 固定三角形, 三角形有且只有一点为超级三角形顶点且该三角形一边为散点凸壳边 $\alpha$ 且该三角形剩下一个顶点和凸壳剩下的所有点不在线 $\alpha$ 同一侧。

\section{3 实例测试}

实验设备处理器配置为 $\operatorname{Interl}(\mathrm{R})$ Pentium(R) CPU G630 2.70GHz, 经测试 361 个散点平均三角化时间为 $24 \mathrm{~ms}$, 如图 9(a)所示。矿井安全监测工程应用中钻孔设备往往仅有数十个, 如图 9(b) 为 23 个测力点的三 角化网格。图 9(c)为设置压力间隔为 20 个时等值线追踪后的图形, 其中白色线条为等值线。图 9(d)为设置 压力间隔为 1000 个时生成的“云图”。

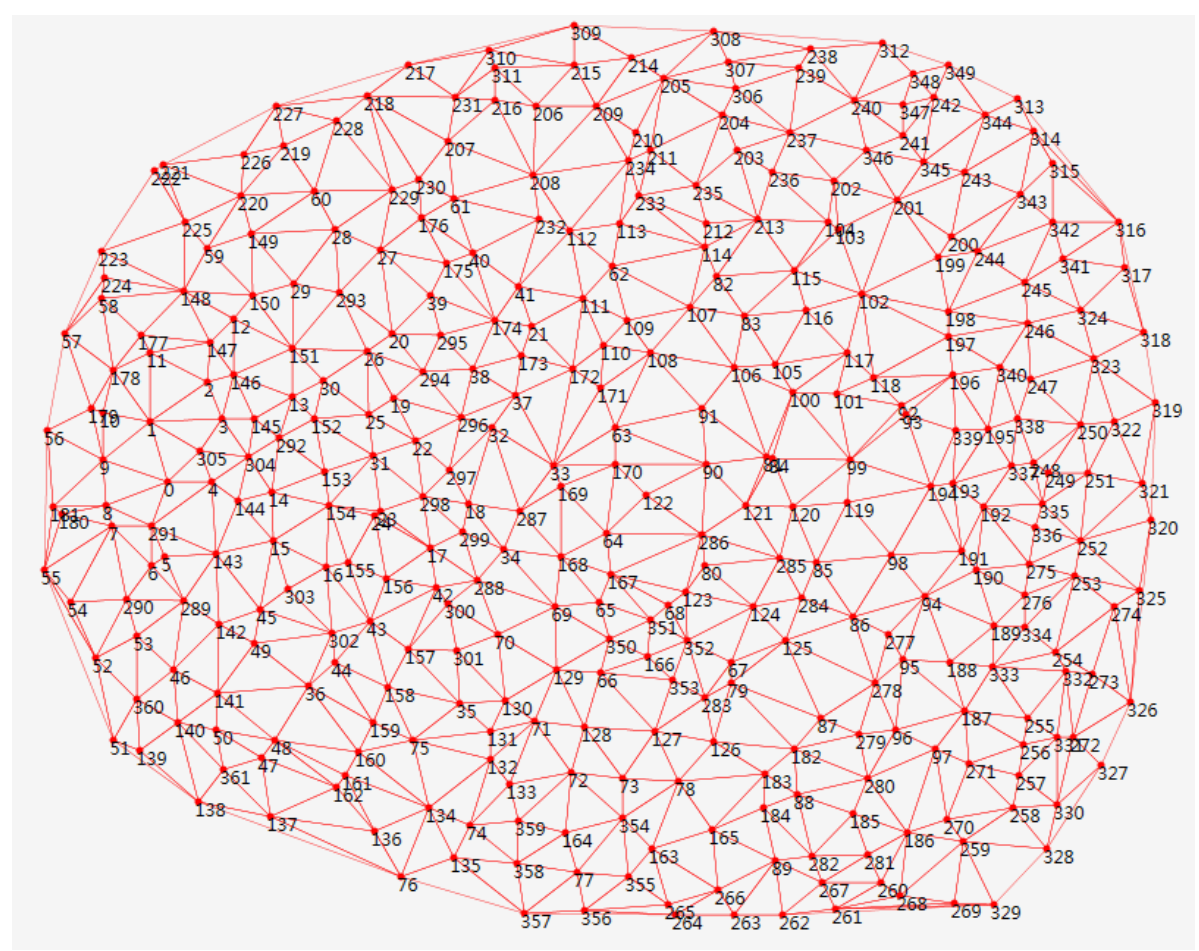

(a)

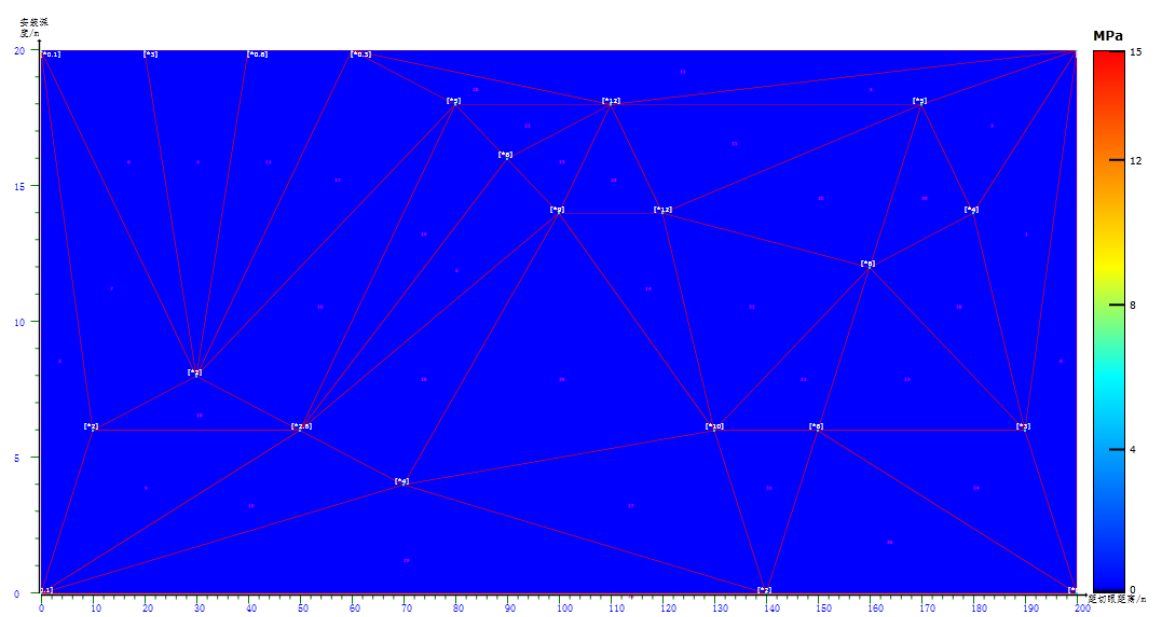

(b) 


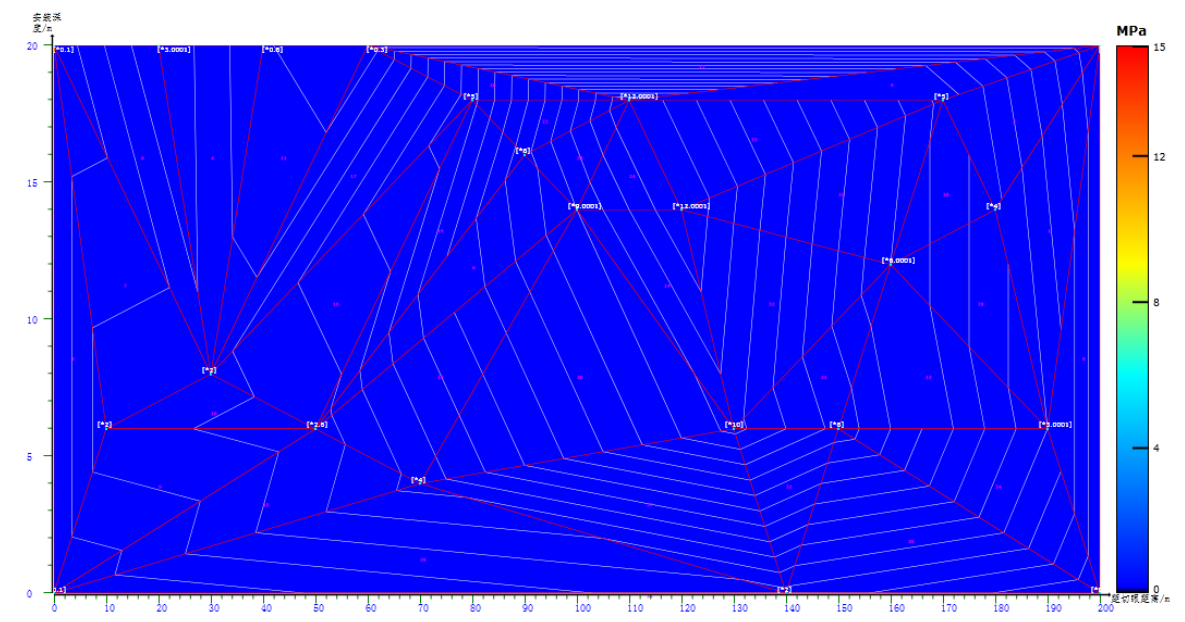

(c)

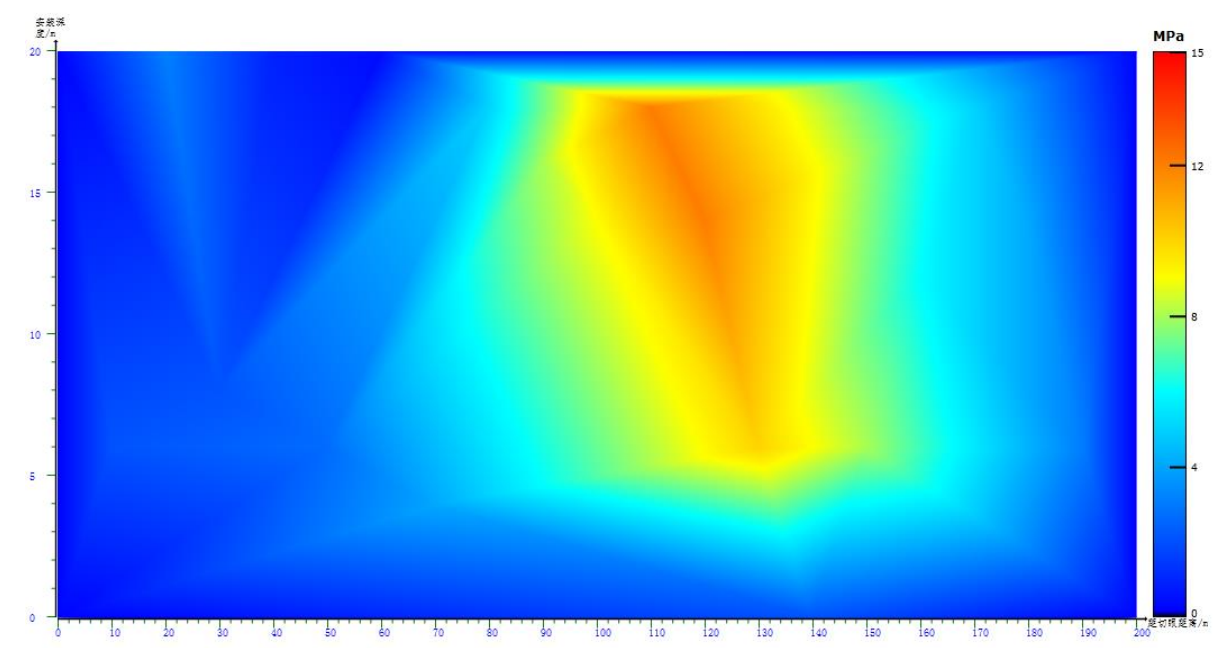

(d)

图 9 云图生成示意图

\section{4 总结与展望}

Bowyer-Watson 常用算法存在无法形成三角网和凸壳边丢失的问题, 基于 Delaunay 最优性原则的局部 优化过程 LOP 可解决上述问题, 但其引发的对邻接三角形维护所带来的空间消耗和时间复杂度降低了算法 效率。本文设计了一种基于点-邻接点-三角形的 $\mathrm{O}(\mathrm{nlgn})$ 三角化算法, 并实现了等值线追踪和等值域填充 功能, 在提高效率的同时解决了三角形丢失问题。本文只对单一分块的数据进行算法设计, 下一步我们将 针对实际工程应用中大量离散点情况下的算法优化及应用展开相关研究。

\section{参考文献:}

[1] 孙桂茹, 马亮, 陆登平, 赵国瑞, 郝嘉霖. 等值线生成与图形填充算法. 天津大学学报, 2000, 33 (6)：816-818.

[2] 蒋瑜, 杜斌, 卢军, 等. 基于 Delaunay 三角网的等值线绘制算法 [J]. 计算机应用研究, 2010, 27 (1)：101-103.

[3] A. Bowyer, Computing Dirichlet tessellations, The Computer J., 24:2(1981), 162-166.

[4] D.F. Watson, Computing the n-dimensional Delaunay tessellation with Application to Voronoi polytopes, The Comuter J., 24: 2(1981), 167-172.

[5] 宋占峰, 蒲浩, 詹振炎. 基于三角网数字地面模型快速定位算法的研究 [J]. 中国铁道科学, 2002, 23(1) :63-66.

[6] Zhou Sheng, Jones C B. HCP0: an efficient insertion order for incremental Delaunay triangulation[J]. Information Processing Letters, 2005, $93(1): 37-42$. 
[7] 向传杰, 朱玉文. 一种高效的 Delaunay 三角网合并生成技术 $[J]$. 计算机应用，2002, 22 (11):34-36.

[8] 吴宇晓, 张登荣. 生成 Delaunay 三角网的快速合成算法 [J]. 浙江大学学报, 2004, 31 (3)：343-348.

[9] 邬吉明, 沈隆均, 张景琳. Delaunay 三角网格的一种快速生成法 [J]. 数值计算与计算机应用, 2001, 12 (4) : 267-275.

[10] G. L. Dirichlet, Uber die Reduction der Positiven Quadratischen Formen mit drei Unbestimmten Ganzen Zahlen, Z. Reine Angew. Math., 40:3(1850), 209-227.

\section{作者简介:}

丁扬 (1985 年- ), 男, 汉族, 山东泰安人, 工程师, 工程硕士学位, 研究方向为计算机网络, 计算数学。

郗富琦（1984 年- ), 男, 汉族, 山东泰安人, 学士学位, 研究方向为煤矿安全监测。

杨祥来 (1986 年- ), 男, 汉族, 山东泰安人, 工程师, 硕士学位, 研究方向为云计算, 大数据分析。

联系作者: 丁扬, Emai1: jordandy@163.com, 15588595387

\section{References:}

[1] Sun Guiru, Ma Liang, Lu Dengping, Zhao Guorui, Hao Jialin. Contour Generation and Filling Graph Algorithm .Journal of Tianjin University,2000,33(6):816-818.

[2] Jiang Yu,Du Bin, Lu June, and so on. Contour Drawing based on Delaunay Triangulation Algorithm [J].Application Research of Computers,2010,27(1):101-103.

[3] A. Bowyer, Computing Dirichlet tessellations, The Computer J., 24:2(1981), 162-166.

[4] D.F. Watson, Computing the n-dimensional Delaunay tessellation with Application to Voronoi polytopes, The Comuter J., 24: 2(1981), 167-172.

[5] Song Zhanfeng, Pu Hao, Zhan Zhenyan. Fast Locating Algorithm Research Based on Triangle Digital Terrain Model [J].China Railway Science,2002,23(1):63-66.

[6] Zhou Sheng, Jones C B.HCPO: an efficient insertion order for incremental Delaunay triangulation[J]. Information Processing Letters, 2005,93(1):37-42.

[7] Xiang Chuanjie, Zhu Yuwen. Consolidation Generation Technology of Delaunay Triangulation with High Efficient [J].Application Research of Computers,2002,22(11):34-36.

[8] Wu Yuxiao, Zhang Dengrong. Rapid Synthesis Algorithm to Generate Delaunay Triangulation [J].Journal of Zhejiang University,2004,31(3):343-348.

[9] Wu Jiming, Shen Longjun, Zhang Jinglin. A Fast Generation Method for Delaunay Triangulations [J].Journal on Numerical Methods and Computer Applications,2001,12(4):267-275.

[10] G.L. Dirichlet, Uber die Reduction der Positiven Quadratischen Formen mit drei Unbestimmten Ganzen Zahlen, Z.Reine Angew. Math., 40:3(1850), 209-227.

\section{About author:}

Ding Yang (born in 1985): male with Han nationality from Taian, Shandong as as engineer; gained master's degree in engineering with computer networks and computational mathematics as his research direction.

Xi Fuqi (born in 1984): male with Han nationality from Taian, Shandong; gained master's degree with safety supervision in coal mine as his research direction.

Yang Xianglai (born in 1986): male with Han nationality from Taian, Shandong as as engineer; gained master's degree in engineering with cloud computing and big data analytics as his research direction.

Contact author: Ding Yang,Email:jordandy@ 163.com,15588595387 